\title{
ERROR ESTIMATES FOR FINITE DIFFERENCE APPROXIMATIONS TO HYPERBOLIC EQUATIONS FOR LARGE TIME
}

\author{
WILLIAM LAYTON ${ }^{1}$
}

\begin{abstract}
Convergence results for bounded time intervals are well known for finite difference approximations to the Cauchy problem for hyperbolic equations. These results typically state that if the initial data is smooth and the approximation is stable in $L^{2}$ and accurate of order $r$, then the error at time $t$ is bounded by $C(t, f) h^{r}$, where $f$ is the initial data and $C(t)=O(t)$.

This paper considers the error for long times. It is not possible for the error to be $O\left(h^{r}\right)$ in $L^{p}$ uniformly in $t$. However, it is shown here that if $\Omega$ is a bounded domain the error in $L^{p}(\Omega)$ is bounded by $C(\Omega, f) h^{r}$, where $C$ is independent of $t$. Thus, the global error will grow as more timesteps are taken but the local error will remain uniformly bounded.
\end{abstract}

1. Introduction. Consider a finite difference approximation

$$
\begin{aligned}
& v_{n+1}(x)=A v_{n}(x), \quad v_{0}(x)=f(x), \quad-\infty<x<\infty, \\
& A v(x)=\sum_{j \in \mathbf{Z}} a_{j} v(x+j h), \quad \sum_{j \in \mathbf{Z}}\left|a_{j}\right|<\infty,
\end{aligned}
$$

to the initial value problem for $u=u(x, t)$

$$
\frac{\partial u}{\partial t}=\frac{\partial u}{\partial x}, \quad u(x, 0)=f(x), \quad-\infty<x<\infty, t \geq 0 .
$$

In (1.1), $h, k>0, k / h=\lambda \equiv$ const, and $v_{n}(x)$ is the approximation to $u(x, n k)$. The difference approximation (1.1) is assumed to be stable in $L^{2}$ and accurate of order $r$.

The convergence of schemes such as (1.1) to the solution of (1.2) is a classical problem in numerical analysis and has been studied by many people. As an example, consider the following standard result that can be found in many places, for example Theorem 4.2 of Peetre and Thomée [5] (with $p=2$ ).

THEOREM 1.1. Assume $f \in W_{2}^{r+1}(\mathbf{R})$. Then, given a $T>0$ there is a $C=$ $C(T)$ such that for $t_{n}=n k \leq T$

$$
\left\|u\left(x, t_{n}\right)-v_{n}(x)\right\|_{L^{2}(\mathbf{R})} \leq C(T) h^{r}\|f\|_{W_{2}^{r+1}} .
$$

This is a very sharp and satisfactory convergence result in every way except one. The solution to the difference equation (1.1) and the differential equation (1.2) can be shown to be uniformly bounded in $t$ in the $L^{2}(\mathbf{R})$ norm. However, the constant

Received by the editors January 29, 1983 and, in revised form, June 29, 1983, August 25, 1983 and February 17, 1984.

1980 Mathematics Subject Classification. Primary 65M15; Secondary 35L45.

Key words and phrases. Finite difference method, hyperbolic equation, uniform error estimate.

${ }^{1}$ Research partially supported by the National Science Foundation under grant MCS-8202025.

(C) 1984 American Mathematical Society $0002-9939 / 84 \$ 1.00+\$ .25$ per page 
in the error estimate grows, $C(T)=C \cdot T$. Analogous results to Theorem 1.1 hold in other $L^{p}(\mathbf{R})$ norms, but in all of them the constants involved increase with $T$, $C(T)=O(T)$.

In the above theorem the constant $C(T)$ cannot be replaced by an absolute constant independent of $T$. However, it will be shown here that if $\Omega \subset \mathbf{R}$ is a bounded set then an estimate of the form (1.3) holds in $L^{p}(\Omega)$ with $C$ independent of $T$ :

$$
\left\|u\left(\cdot, t_{n}\right)-v_{n}(\cdot)\right\|_{L^{p}(\Omega)} \leq C h^{r}, \quad C=C(\Omega, f) .
$$

The exact dependence of $C$ on $\Omega$ and the smoothness of $f$ will be given in the statement of the theorem. Thus, although the error in (1.1) might increase in $t$ globally, locally the error is uniformly bounded in $t$.

Let $a(\theta)$ denote the symbol of the difference operator $A$,

$$
a(\theta)=\sum_{j \in \mathbf{Z}} a_{j} e^{i j \theta} .
$$

Then, it is well known that stability in $L^{2}(\mathbf{R})$ is equivalent to the condition $|a(\theta)| \leq$ 1 for $|\theta| \leq \pi$. If $|a(\theta)| \equiv 1$ then the approximation (1.1) is conservative. The approximation (1.1) is dissipative if $|a(\theta)|<1$ except for a finite number of points.

The difference scheme (1.1) is accurate of order $r>0$ if, for every sufficiently smooth solution of (1.2),

$$
u(x,(n+1) k)=A u(x, n k)+O\left(h^{r+1}\right) .
$$

Also, it is well known that (1.1) is accurate of order $r$ if and only if

$$
a(\theta)=\exp (i \lambda \theta[1+\phi(\theta)])
$$

where $\phi(\theta)=O\left(\theta^{r}\right)$ as $|\theta| \rightarrow 0$. For a proof of this see Thomée [8].

The main result of this paper is

THEOREM 1.2. Assume (1.1) is stable in $L^{2}$ and accurate of order $r$. Let $\Omega \subset \mathbf{R}$ be a bounded domain with midpoint $\bar{x}$. Then, there is a constant independent of $h, f, x$ and $t$ such that for every $t=n k \geq 0$

$$
\left\|u(\cdot, t)-v_{n}(\cdot)\right\|_{L^{2}(\Omega)} \leq C h^{r}\left\{\operatorname{diam}(\Omega)\|f\|_{W_{2}^{r+1}}+\|(x-\bar{x}) f(x)\|_{W_{2}^{r+1}}+\|f\|_{W_{2}^{r}}\right\} .
$$

In the above the Sobolev norms are over $\mathbf{R}$, i.e., $W_{2}^{s}(\mathbf{R})$. Combining the technique of proof of the above with the method of obtaining $L^{\infty}$ estimates introduced in [3] gives a pointwise bound that is uniform in $t$. (Thus, uniform in $t, O\left(h^{r}\right)$ errors in $L^{p}(\Omega)$ follow for every $p$ and every bounded subdomain $\Omega$.)

THEOREM 1.3. Assume (1.1) is stable in $L^{2}$ and accurate of order $r$. Then, there is a constant $C$ independent of $h, f, x$ and $t$ such that for every $t=n k \geq 0$

$$
\left|u(x, t)-v_{n}(x)\right| \leq C h^{r}\left\{|x| \cdot\|f\|_{0, r+2,1}+\|f\|_{1, r+1,1}\right\} .
$$

Here the norms $\|\cdot\|_{m, s, p}$ are a measure of the smoothness of $f$ in the transform space and will be introduced in the next section. The smoothness conditions on $f$ are trivially satisfied if, for example, $f \in S$, the Schwartz space of $C^{\infty}$ rapidly decreasing functions. 
2. Notation and preliminaries. For $1 \leq p \leq \infty, L^{p}(\mathbf{R})$ denotes the usual $L^{p}$ space of Lebesgue measurable functions $f: \mathbf{R} \rightarrow \mathbf{C}$. Define the Fourier and inverse Fourier transform of a function $f(x), g(\theta)$ as

$$
\begin{aligned}
& (\mathcal{F} f)(\theta)=\hat{f}(\theta)=(2 \pi)^{-1 / 2} \int_{\mathbf{R}} f(x) e^{-i x \theta} d \theta, \\
& \left(\mathcal{F}^{-1} g\right)(x)=\check{g}(x)=(2 \pi)^{-1 / 2} \int_{\mathbf{R}} g(\theta) e^{i x \theta} d \theta .
\end{aligned}
$$

The smoothness conditions on $f$ are stated in the transform space. Define, e.g. following Chapter 9 of Nikol'skii [4], the Bessel-MacDonald potential operator $I_{s}$,

$$
I_{s} u=\mathcal{F}^{-1}\left(1+|\theta|^{2}\right)^{-s / 2}(\mathcal{F} u)(\theta), \quad-\infty<s<\infty .
$$

$I_{s} u$ can be written in the $x$ (untransformed) variables as convolution of $u$ with the Bessel-MacDonald kernel. Define the norm $\|\cdot\|_{m, s, p}$ by

$$
\|u\|_{m, s, p}^{p}=\sum_{j=0}^{m}\left\|\mathcal{F}\left(I_{-s}\left(x^{j} u\right)\right)\right\|_{L^{p}(\mathbf{R})}^{p},
$$

with the usual modification if $p=\infty$. In $\|\cdot\|_{m, s, p}, s$ is the degree of smoothness, $m$ is the decay at infinity and $p$ is the $L^{p}$ norm in which both are measured.

In particular, note that for all $m, s, p<\infty$,

$$
\|u\|_{m, s, p}=\left[\sum_{j=0}^{m} \int_{\mathbf{R}}\left(1+|\theta|^{2}\right)^{p s / 2}\left|\frac{d^{j}}{d \theta^{j}} \hat{u}(\theta)\right|^{p} d \theta\right]^{1 / p}
$$

and when $p=2, s \in \mathbf{Z}^{+}$,

$$
\|u\|_{m, s, p}^{2}=\|u\|_{W_{2}^{s}(\mathbf{R})}^{2}+\|x u\|_{W_{2}^{s}(\mathbf{R})}^{2}+\cdots+\left\|x^{m} u\right\|_{W_{2}^{s}(\mathbf{R})}^{2} .
$$

Convolution in the $\theta$ variable will always be denoted by $*$. Note that if $\chi \in S$, then $\hat{\chi} * \hat{f} \in S$ and that

$$
d(\hat{\chi} * \hat{f}) / d \theta=\hat{\chi}^{\prime} * \hat{f}=\hat{\chi} * \hat{f}^{\prime}
$$

3. Proof of the theorems. First note that since the difference and the differential equation are translation invariant it is sufficient to prove Theorem 1.2 in the case $\Omega=[-R, R]$ with $\bar{x}=0$. Let $\hat{u}(\theta, t), \hat{v}_{n}(\theta)$ denote the Fourier transforms of the solutions of $(1.1),(1.2)$ respectively and $e\left(x, t_{n}\right)=u\left(x, t_{n}\right)-v_{n}(x)$ the error. $\hat{e}(\theta, t)$ then satisfies

$$
\hat{e}(\theta, t)=\left[\exp (t i \theta)-a(h \theta)^{n}\right] \hat{f}(\theta) .
$$

Let $\chi \in C_{0}^{\infty}$ be a cutoff function for $\Omega$, i.e., $\chi \equiv 1$ on $\Omega$ and $\chi=0$ outside a neighborhood of $\Omega$. Then,

$$
\|e(\cdot, t)\|_{L^{2}(\Omega)} \leq\|\chi(\cdot) e(\cdot, t)\|=\|\hat{\chi} * \hat{e}\| .
$$

Define the functions

$$
P(\theta, t, h)=(1-\exp (t i \theta \phi(h \theta))) \hat{f}(\theta), \quad Q(\theta, t, h)=\partial P(\theta, h, t) / \partial \theta .
$$


Using (1.4) in (3.1), (3.2) gives

$$
\begin{aligned}
\|e(\cdot, t)\|_{L^{2}(\Omega)} & \leq\left\|\hat{\chi} *\left(e^{t i \theta} P(\theta, t, h)\right)\right\| \\
& \leq\left\|\frac{\partial}{\partial \theta}\left(\hat{\chi}^{*}\left(\frac{e^{t i \theta}}{i t} P(\theta, t, h)\right)\right)-\hat{\chi} *\left(\frac{e^{t i \theta}}{i t} Q(\theta, t, h)\right)\right\| .
\end{aligned}
$$

In the above the differentiations with respect to $\theta$ are legitimate since $\hat{\chi} * \hat{e} \in \mathcal{S}$. Using the triangle inequality in the above and making a change of variables in the first convolution gives

$$
\|e\|_{L^{2}(\Omega)} \leq \frac{1}{t}\left\|\hat{\chi}^{\prime} *\left(e^{t i \theta} P(\theta, t, h)\right)\right\|+\frac{1}{t}\left\|\hat{\chi} *\left(e^{t i \theta} Q(\theta, t, h)\right)\right\| .
$$

Convolution with $\hat{\chi}$ is a bounded linear operator with norm $\sup _{x}|\chi(x)|=1$ and convolution with $\hat{\chi}^{\prime}$ has operator norm

$$
1+\sup _{x \in \mathbf{R}}|x \chi(x)|=C \operatorname{diam}(\Omega) .
$$

Thus, we arrive at

$$
\|e(\cdot, t)\|_{L^{2}(\Omega)} \leq C t^{-1} \operatorname{diam}(\Omega)\|e(\cdot, t)\|+t^{-1}\left\|e^{t i \theta} Q(\theta, t, h)\right\| .
$$

The first term in the above inequality is the quantity bounded in Theorem 1.1, with the exception that the $t^{-1}$ cancels the $t$ in the estimate in Theorem 1.1. This gives the first term occurring in the error estimate in Theorem 1.2.

Next, consider the second term in the above by expanding $\partial P(\theta, t, h) / \partial \theta$ :

$$
\begin{aligned}
t^{-1}\left\|e^{t i \theta} Q(\theta, t, h)\right\| \leq & t^{-1}\left\|\left[e^{t i \theta}-a(h \theta)^{n}\right] \hat{f}^{\prime}(\theta)\right\| \\
& +t^{-1}\left\|t\left[\phi(h \theta)+h \theta \phi^{\prime}(h \theta)\right] \hat{f}(\theta)\right\| .
\end{aligned}
$$

In the second term in the above, the fact that all the exponentials are $\leq 1$ in modulus was used. The first term in the preceding inequality is the error in the scheme with initial data $x f(x)$. The argument in the proof of Theorem 1.1 gives

$$
t^{-1}\left\|\left[e^{t i \theta}-a(h \theta)^{n}\right] \hat{f}^{\prime}(\theta)\right\| \leq C h^{r}\|x f(x)\|_{W_{2}^{r+1}} .
$$

Finally, it will be shown that the last integral in (3.4) is $O\left(h^{r}\right)$ uniformly in $t$. To this end, consider $\phi(\theta)$. $\phi(\theta)$ can be calculated explicitly from the consistency condition

$$
\phi(\theta)=(i \lambda \theta)^{-1} \ln a(\theta)-1 .
$$

Note that $a(\theta)$ is a smooth periodic function of $\theta$ that is bounded away from zero so that the above is well defined. From the above,

$$
\phi(\theta)+\theta \phi^{\prime}(\theta)=(i \lambda a(\theta))^{-1} a^{\prime}(\theta)-1 .
$$

Thus, $\phi(\theta)+\theta \phi^{\prime}(\theta)$ is uniformly bounded in $\theta$. Since $\phi$ is smooth, as $|\theta| \rightarrow 0$

$$
\left|\phi(\theta)+\theta \phi^{\prime}(\theta)\right| \leq C|\theta|^{r} .
$$

Now, consider the last term in (3.4). By the triangle inequality, (3.6) and the above remarks

$$
\begin{aligned}
\left\|\left(\phi(h \theta)+h \theta \phi^{\prime}(h \theta)\right) \hat{f}(\theta)\right\|^{2} \leq & \int_{|\theta| \leq \pi h^{-1}}\left|\phi(h \theta)+h \theta \phi^{\prime}(h \theta)\right|^{2}|\hat{f}(\theta)|^{2} d \theta \\
& +C \int_{|\theta|>\pi h^{-1}}|\hat{f}(\theta)|^{2} d \theta \\
\leq & C h^{2 r}\|f\|_{W_{2}^{r}}^{2}+C \int_{|\theta|>\pi h^{-1}}|\hat{f}(\theta)|^{2} d \theta .
\end{aligned}
$$


For $|\theta|>\pi h^{-1},\left(\pi h^{-1}\right)^{-2 r}\left(1+|\theta|^{2}\right)^{r} \geq 1$. Thus,

$$
\begin{aligned}
\int_{|\theta|>\pi h^{-1}}|\hat{f}(\theta)|^{2} d \theta & \leq \pi^{-2 r} h^{2 r} \int_{\mathbf{R}}\left(1+|\theta|^{2}\right)^{r}|\hat{f}(\theta)|^{2} d \theta \\
& \leq \pi^{-2 r} h^{2 r}\|f\|_{W_{2}^{r}}^{2}
\end{aligned}
$$

Using the previous inequality and (3.4)-(3.7) in (3.3) gives Theorem 1.2.

The pointwise bound in Theorem 1.3 follows by applying the Fourier inversion formula in (3.1):

$$
e(x, t)=(2 \pi)^{-1 / 2} \int_{\mathbf{R}} e^{t i \theta}\left[e^{i x \theta}(1-\exp (t i \theta \phi(h \theta))) \hat{f}(\theta)\right] d \theta .
$$

Next, this is integrated by parts directly, bringing down a $|t|^{-1}$ factor. Then, the pointwise estimate follows by the technique introduced in [5] and modifying, where appropriate, the argument in the case of the $L_{\text {loc }}^{2}$ estimates.

4. Two examples. In this section two examples of numerical schemes for (1.2) are considered. The first is a generic, implicit, two-level method. The aims in the first example are simply to point out that implicit methods can formally be put in the form of $(1.1)$ and to look at the smoothness conditions on $a(\theta)$ that are satisfied by typical difference schemes. The second example is the simplest method-of-lines approximation to (1.2). Once again, this approximation can formally be expressed in the form (1.1). Further, in this scheme the function $\phi(\theta)$ can be computed exactly and the conditions of Theorem 1.2 verified explicitly. A two-level difference scheme for (1.2) can be written in the form

$$
A_{1} v_{n+1}(x)=A_{2} v_{n}(x), \quad v_{0}(x)=f(x),
$$

where

$$
A_{1} w(x)=\sum_{\substack{j \in \mathbf{Z} \\ \text { (finite sum) }}} \alpha_{j} w(x+j h), \quad A_{2} w(x)=\sum_{\substack{j \in \mathbf{Z} \\ \text { (finite sum) }}} \beta_{j} w(x+j h) .
$$

The symbol of the difference scheme (4.1) is then $a(\theta)=a_{1}(\theta)^{-1} a_{2}(\theta)$, where

$$
a_{1}(\theta)=\sum_{j \in \mathbf{Z}} \alpha_{j} e^{i j \theta}, \quad a_{2}(\theta)=\sum_{j \in \mathbf{Z}} \beta_{j} e^{i j \theta} .
$$

The difference operator $A_{1}$ is invertible if and only if $a_{1}(\theta) \neq 0$ for all $\theta$. Thus, $a(\theta)$ is a meromorphic function of $z=e^{i \theta}$ that is periodic in $\theta$ and uniformly bounded on $\theta \in \mathbf{R}$.

Expanding $a(\theta)$ in a Fourier series, $a(\theta)=\sum_{j \in \mathbf{Z}} a_{j} e^{i j \theta}$, the implicit method (4.1) is easily seen to be equivalent to an explicit method of the form (1.1) with

$$
A w(x)=\sum_{j \in \mathbf{Z}} a_{j} w(x+j h)
$$

Fully-discrete finite element methods for (1.2) can be written in the form (4.1) also. Thus, Theorem 1.2 applies for finite element methods as well.

The simplest continuous-in-time scheme for (1.2) arises by replacing the derivative $\partial / \partial x$ by the central difference approximation. Thus, $v(x, t)$ approximates $u(x, t)$ and satisfies the equations

$$
\frac{d}{d t} v(x, t)=\frac{v(x+h, t)-v(x-h, t)}{2 h}, \quad v(x, 0)=f(x) .
$$


The transform of the above equation is

which has solution

$$
\frac{d}{d t} \hat{v}(\theta, t)=\frac{i \sin (\theta h)}{h} \hat{v}(\theta, t), \quad \hat{v}(\theta, 0)=\hat{f}(\theta)
$$

$$
\hat{v}(\theta, t)=\exp \left(t^{-1} i \sin (h \theta)\right) \hat{f}(\theta) .
$$

Expanding the $C^{\infty}$ periodic function in a Fourier series $(\lambda=k / h \equiv$ const $)$

$$
\exp (\lambda i \sin \theta)=\sum_{j \in \mathbf{Z}} a_{j} e^{i j \theta}
$$

it is clear that the method of lines approximation (4.1) is equivalent to a finite difference method

$$
v_{n+1}(x)=\sum_{j \in \mathbf{Z}} a_{j} v(x+j h), \quad v_{0}(x)=f(x),
$$

of the form (1.1) where the finite difference operator has symbol $a(\theta)=\exp (\lambda i \sin \theta)$. Clearly,

$$
a(\theta)=\exp (i \lambda \theta[1+\phi(\theta)]), \quad \phi(\theta)=(\sin \theta) / \theta-1,
$$

so that $\phi(\theta)=O\left(\theta^{2}\right)$ as $\theta \rightarrow 0$ and (4.1) is indeed a second order method. As for $\phi(\theta)+\theta \phi^{\prime}(\theta)$, consider $\phi^{\prime}(\theta)$ :

$$
\phi^{\prime}(\theta)=\frac{1}{\theta}(\cos \theta-\sin \theta / \theta) .
$$

A straightforward Taylor series calculation gives that $\phi^{\prime}(\theta)=O(\theta)$ as $\theta \rightarrow 0$. Also, $\phi(\theta)+\theta \phi^{\prime}(\theta)$ is uniformly bounded. In fact,

$$
\left|\phi(\theta)+\theta \phi^{\prime}(\theta)\right|=|\cos \theta-1| \leq 2 .
$$

5. Remarks. It is easiest to see that an error estimate uniform in $t$ cannot hold globally when the method (1.1) is dissipative. In this case, the dominated convergence theorem implies that $v_{n}(x) \rightarrow 0$ in $L^{2}(\mathbf{R})$ as $n \rightarrow \infty$. Since $\|u(\cdot, t)\|=$ $\|f\|$ it follows that the error in $L^{2}(\mathbf{R})$ must be $O(1)$ for large $t$. That is, the constant $C(T)$ in Theorem 1.1 cannot be replaced by a constant independent of $T$.

The dissipative case also sheds some light on the necessity of using the triply indexed norms in Theorem 1.3, and the requirement of decay at infinity in the initial data in Theorem 1.2. Indeed, it is not possible for an inequality of the form

$$
\left\|u\left(\cdot, t_{n}\right)-v_{n}\right\|_{L^{2}(\Omega)} \leq C h^{r}\|f\|_{W_{2}^{s}}
$$

to hold with $C$ independent of $t$ for any $s, 0 \leq s<\infty$. Specifically, (5.1) implies the condition

$$
\sup _{t_{n} \geq 0} \sup _{f \in W_{2}^{s}} \frac{\left\|u\left(\cdot, t_{n}\right)-v_{n}\right\|_{L^{2}(\Omega)}}{\|f\|_{W_{2}^{s}}} \leq C h^{r} .
$$

If the scheme is dissipative then $v_{n} \rightarrow 0$ as $n \rightarrow \infty$ and the previous equation holds if

$$
\sup _{f \in W_{2}^{s}} \frac{\left\|u\left(\cdot, t_{n}\right)\right\|_{L^{2}(\Omega)}}{\|f\|_{W_{2}^{s}}} \rightarrow 0, \quad \text { as } t_{n} \rightarrow \infty .
$$

If the support of $f$ is $\Omega-t=\{x \mid x+t \in \Omega\}$, then the support of $u$ at time $t$ will be in $\Omega$ so that the above quotient will be bounded below for all time by a positive constant.

Thus, (5.1) cannot hold and it is critical that the norms in Theorem 1.2 include measures of the decay at $\pm \infty$ of $f$. 


\section{REFERENCES}

1. G. S. Ávila and D. G. Costa, Asymptotic properties of general symmetric hyperbolic systems, J. Funct. Anal. 35 (1980), 49-63.

2. P. D. Lax and R. S. Phillips, Scattering theory, Academic Press, New York, 1967.

3. W. Layton, Simplified $L^{\infty}$-estimates for difference approximation to partial differential equations, Proc. Amer. Math. Soc. 86 (1982), 491-495.

4. S. M. Nikol'skii, Approximation of functions of several variables and embedding theorems, SpringerVerlag, New York, 1975.

5. J. Peetre and V. Thomée, On the rate of convergence for discrete initial-value problems, Math. Scand. 21 (1967), 159-176.

6. R. D. Richtmeyer and K. W. Morton, Difference methods for initial value problems, 2nd ed., Interscience, New York, 1967.

7. G. Strang, Accurate partial difference methods. I. Linear Cauchy problems, Arch. Rational Mech. Anal. 12 (1963), 392-402.

8. V. Thomée, Stability of difference schemes in the maximum norm, J. Differential Equations 1 (1965), 273-292.

\section{Mathematics Institute, Catholic University, NiJmegen, The Netherlands}

Current address: School of Mathematics, Georgia Institute of Technology, Atlanta, Georgia 30332 